\title{
Structure of the Universe
}

Paul T E Cusack*

BScE, DULE, 23 Park Ave, Saint John, NB E2J 1R2, Canada

\section{Article Info}

\section{*Corresponding author:}

Paul T E Cusack

BScE, DULE

23 Park Ave

Saint John, NB E2J 1R2

Canada

Tel: +1-506-652-6350

E-mail: St-michael@hotmail.com

Received: November 17, 2018

Accepted: November 27, 2018

Published: January 3, 2019

Citation: Cusack PTE. Structure of the Universe. Int J Cosmol Astron Astrophys. 2019; 1(1): 4-6. doi: $10.18689 /$ ijcaa-1000102

Copyright: $\odot 2019$ The Author(s). This work is licensed under a Creative Commons Attribution 4.0 International License, which permits unrestricted use, distribution, and reproduction in any medium, provided the original work is properly cited.

Published by Madridge Publishers

\begin{abstract}
In this paper, we consider how the universe may have come to exhibit the structure that it does. Viz uniform distribution on mass throughout and an ellipsoid shape. This finding doesn't agree with previous theories which have the universe as expanding. In fact, the universe is being compressed and mass results.
\end{abstract}

Keywords: Mass; Magnetic Force; Ellipsoid; Universe

\section{Introduction}

In this brief paper, we examine why the universe has mass evenly distributed or whey it is uniform. We consider also why mass terminates at 118 elements. And finally, we consider why the universe may be shaped as an ellipsoid [1].

\section{Uniformity Distributed Mass}

The structure of the universe is said to be rather uniform throughout except for black holes or the like. It is much like raindrops on the surface of a glass window. The S.F. (Superfroce) provides for enough energy to allow for the mass of the periodic table of the elements. Space is formed and the universal moment is embedded into the very structure of the universe (Figure 1).

\section{Raindrops on window}
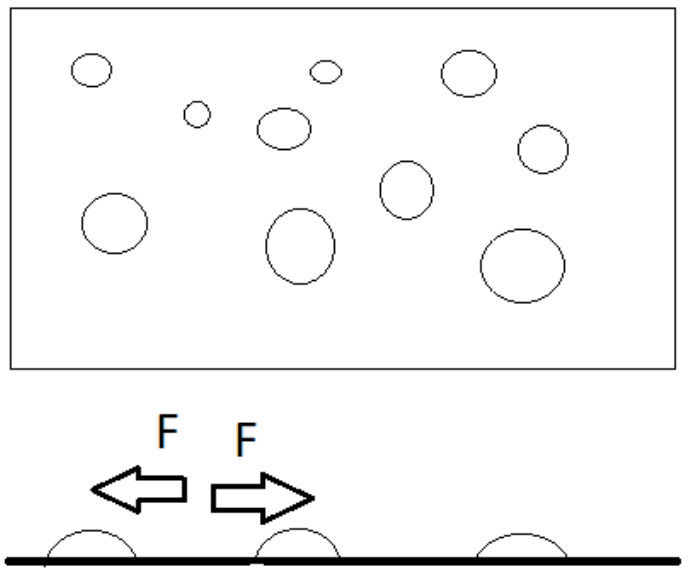

Cross Section

Figure 1. Uniform Mass Distribution

$\mathrm{F}=\mathrm{G} \mathrm{M}_{1} \mathrm{M}_{2} / \mathrm{R}^{2}$

S.F. $=$ Superforce $=8 / 3$

Let $\mathrm{M}_{1}=1$ 
$\mathrm{FR}^{2} /\left[\mathrm{M}_{1} \mathrm{M}_{2}\right]=6.67$

$R^{2} / M_{2}=3998 \sim 0.400 \sim R e$

$\mathrm{R}^{2} / \mathrm{M}=\mathrm{Re}=\mathrm{I} . \mathrm{F} . \mathrm{V} . \mathrm{F}$

$R^{2} / 4.486=402$

$R^{2}=180$

$\mathrm{R}=0.1342$

$R=1-0.8667$

$=0.1334$

=space

$4.482 / 2(1 / \sqrt{ } 2)=1583$

$=$ Moment

$=$ Mom.

$=1-\sin 1$

\section{Magnetic Forces and Mass}

When an experimenter pushes two magnets of like poles together, there is resistance up to a point. Then the resistance is overcome and there is no opposing forces remaining in the bars. It is like that with the universe too. When the resistance of energy being squeezed in to mass is overcome, mass results stored energy (Figure 2).

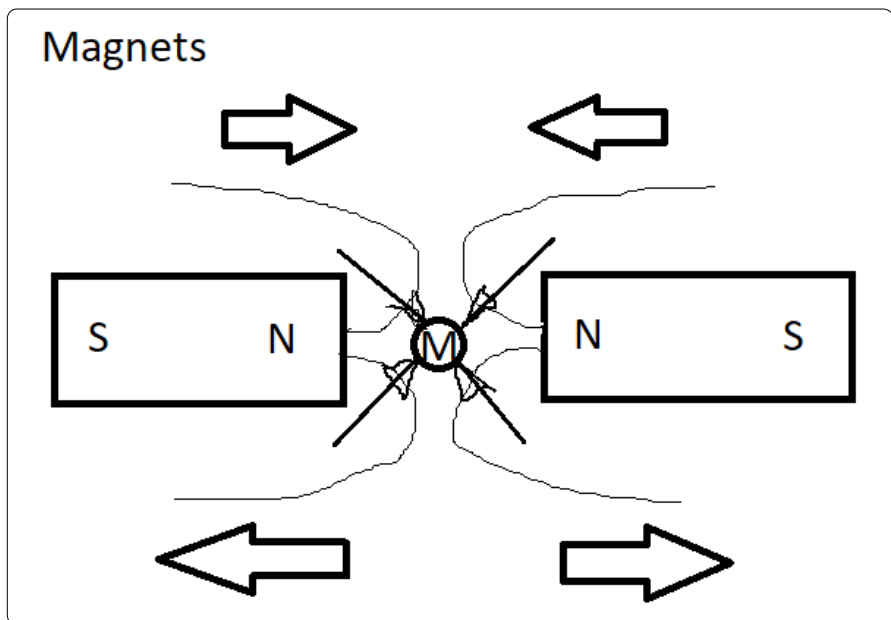

Figure 2. Mass Created by Squeezing Energy

$\mathrm{F}=\mathrm{Ma}$

$\mathrm{F}=8 / 3$

Let $\mathrm{v}=\mathrm{c}=2.9979 \sim 3.0$

$a=1 / \sqrt{ } 2$

Let $M=1$

$\mathrm{Mad} / \mathrm{t}=8$

$M(1 / \sqrt{ } 2) 3=8$

$1 /\{8 \sqrt{ } 2)(3)=1 / M$

$M 1 / M=2.65 \sim S . F$

$265=1 / 4.482 x$

$=118.77=$ Mass of Periodic Table of the Elements.

$\mathrm{F}=\mathrm{Ma}$
$(4,482)(1 / \sqrt{ } 2)$

$=3.169$

$\sim \pi$

$\mathrm{F}=8 / 3=\pi$

$8=3 \pi$

$3 \pi / 8=118.84=$ Mass

\section{Ellipsoid Universe}

The Ellipsoid Universe results from the gravitational forces (Figures 3 and 4). We use the equation for an ellipsoid to yield the gravitational constant.

\section{Elipsoid Universe}

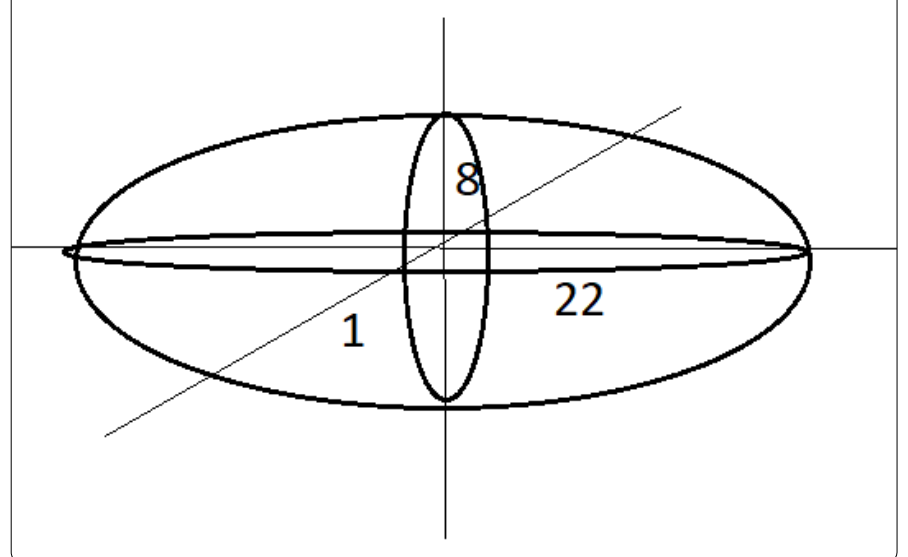

Figure 3. The Ellipsoid Universe takes its shape because of Gravity $66 \times 24 \times 3=1584=1-\sin 1=$ Mom.

$\sqrt{ }\left[1^{2}+8^{2}+22^{2}\right]=23.4=\operatorname{Ln} \pi=\operatorname{Ln} t$

$y=y^{\prime}$

$y=\operatorname{Ln} t$

$y^{\prime}=1 / t=E$

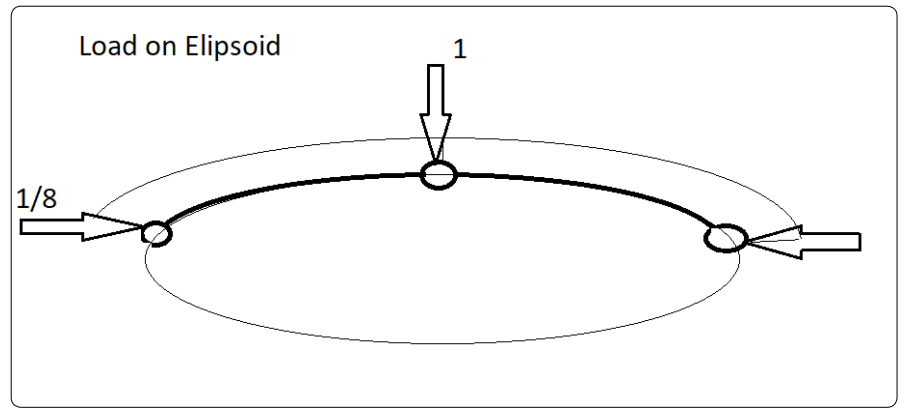

Figure 4. Cross section of the Ellipsoid Universe with a uniform load on the exterior

$\mathrm{d} \theta / \mathrm{dt}=\mathrm{d} \theta / \mathrm{ds}$ where $\mathrm{t}=\mathrm{s}$

$\pi / 2 / 8=\pi / 16=196.3=\infty=19.63 \%$

$\pi / 2 / 22=71.4 \%$

$\operatorname{Ln}(1 / 8)=\operatorname{Lm}(0.125)=$

$0.125=\mathrm{e}^{\mathrm{x}}$

$x=\operatorname{Ln} 0.25$ 
$\mathrm{x}=2.08$

$0.208 \times 19.63=408.3 \sim \operatorname{Re}$

$0.208 \times 71.4 \%=148 \sim 1.5$ Mass Gap

$(1.5 \times 66)+(402 \times 24)=1$ Ellipsoid

Ellipsoid

$\mathrm{x}^{2} / \mathrm{A}^{2}+\mathrm{y}^{2} / \mathrm{B}^{2}=\mathrm{z}^{2} / \mathrm{C}^{2}=1$

$\mathrm{x}=\mathrm{s}=\mathrm{\square}(4 / 3)$

$x^{2} / 24+y^{2} / 66=L x \%=1$

This reduces to:

$4356 x^{2}+576 y^{2}=576(4356)=250.9=$ Period $T$

$\mathrm{T}=1 / \mathrm{t}=\mathrm{E}$

$33561 / E^{2}-1 / E=E-E^{2}$

This reduces to $E^{4}-E^{3}-E+3356=0$

Let $\mathrm{E}=1$

$-1+0.3356=0.664 \sim G=6.67$

\section{Conclusion}

We see that the universe's shape and mass distribution are determined by AT Math considerations.

\section{References}

1. Cusack PTE. Astrotheology, Cusack's Universe. J. Phys. Math. 2016; 7(2): 8. 